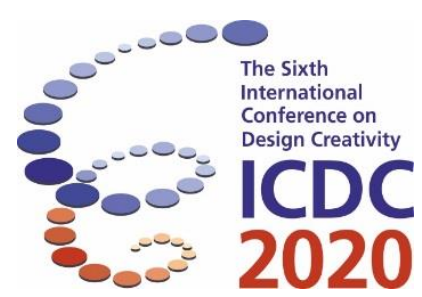

\title{
Semantic measures in design conversations as predictors of creative outcomes in design education
}

\author{
Georgi V. Georgiev ${ }^{1}$ and Hernan Casakin ${ }^{2}$ \\ ${ }^{1}$ Center for Ubiquitous Computing, University of Oulu, Oulu, Finland \\ ${ }^{2}$ School of Architecture, Ariel University, Ariel, Israel
}

\begin{abstract}
The analysis of conversations maintained during the design activity can help to gain a better insight into design thinking and its relation to creativity. A semantic analysis approach was employed to inspect the content of communications and information exchange between students and instructors. The goal was to explore design conversations in terms of Abstraction, Polysemy, Information Content and Semantic Similarity measures, and analyse their relation to the creativity of final design outcomes. These were assessed according to their Originality, Usability, Feasibility, Overall Value and Overall Creativity. To this end, design conversations from the 10th Design Thinking Research Symposium (DTRS10) dataset were used. Main results show a significant relationship between Information Content and Originality and Overall Creativity. For instructors, Semantic measures were mainly related to Feasibility, whereas for students the focus was set on the Overall Value of the final solutions.
\end{abstract}

Keywords: design creativity; design cognition; semantic measures; semantic analysis; creative measures; design education

\section{Introduction}

Design is a most relevant domain for the study of creativity. The analysis of verbal information generated during the design activity can aid in gaining a deeper insight onto creative processes, and creative outcomes. Due to the complexity of such data, a systematic representation is needed to quantify these processes.

Approaches such as semantic analysis can be beneficial to this end, since they allow for a systematic modelling of real-world processes in design problem solving. A characteristic of semantic analysis is that it enables the measurement and comparison of data produced during the design process, and its relation to the design solutions. In spite that an increasing interest has emerged in the last years, not many works quantifying the semantic content of conversations maintained during the design process can be found in literature. Hence, what type of data generated during the design process is associated to the creative outcomes is not evident. Consequently, a study based on the semantic analysis of verbalisations maintained between students and instructors can be beneficial to understand the semantic value of conversations in the design studio, and their relation to design creativity.

The method implemented in this study has several advantages. Firstly, it employs a series of semantic measures allowing the quantification of fundamental phenomena in design, linguistics, and cognitive psychology. These measures demonstrated to be advantageous to investigate ideation in design problem solving, and are concerned with Polysemy, Abstraction, Information Content (IC) and Semantic Similarity (Georgiev and Georgiev, 2018). Secondly, the proposed approach applies systematic and 
domain independent representation of words (i.e., WordNet database). Thirdly, compared to semantic analysis approaches used in the context of design conversations (e.g., Dong, 2009), the studied measures are faster to compute. Hence, the main goal of the present study is to employ the suggested semantic approach to analyse design conversations carried out in the studio, and examine their relation to the creativity of the design outcomes.

Main questions leading the study are: i) how the semantic approach, measured by the Polysemy, Abstraction, IC and Semantic Similarity of the conversations maintained during the design sessions can contribute to analyse the content of the design conversations, and understand its relation to the creativity of the produced outcomes, measured by Originality, Usability, Feasibility, Overall Value and Overall Creativity; and ii) how the relationship between semantic measures and the creativity of the final outcomes differs in regard to students and instructors separately. To address these questions, the industrial design subset of 10th Design Thinking Research Symposium (DTRS10) dataset (Adams and Siddiqui, 2014) is used since it is extensive in number of review sessions, rich in content, and continuous in the process.

\section{Metrics of design creativity}

Creativity has been recognized as an essential component of design. Whereas no comprehensive theory of design creativity can be found in literature so far, it is possible to identify various studies focusing on the evaluation of design creativity. When the assessment of design creativity is concerned with the produced outcome, studies often operationalized it in terms of originality, usability, feasibility, and value. Originality, known as one of the central features of creativity, indicates the extent to which a product differs from other more familiar ones. Originality is defined by Guildford (1981) in terms of the statistical rareness of the outcome. Independently of their novelty, creative designs can also be valuable and useful (Sarkar and Chakrabarti, 2011). Useful, designs must respond to basic requirements and needs, as specified by the task (Siang et al., 2018). Usability can be understood in regard to efficiency, performance, and user satisfaction. To be valuable, design products should be seen by society to worth some merit. Furthermore, in order to be feasible, creative designs might not remain as just creative ideas but should potentially be materialized in real practice (Kreitler and Casakin, 2009). In the present study, the creativity metrics described above are used to assess design outcomes produced by students.

\subsection{Design education and the assessment of creativity}

The promotion and stimulation of creativity is an implicit aim in the design studio (Boucharenc, 2006). In this environment, students acquire theoretical and practical knowledge while they generate concepts and ideas for their design products (Cross, 1983). During the design sessions, also known as design reviews, students learn to think and behave as a professional designer while they develop idea solutions and reflect upon the creativity of their outcomes (Christensen and Ball, 2016). Instructors, on the other hand, evaluate and criticize the produced ideas and outcomes, suggesting changes and actions to be taken over the design (Demirbas and Demirkan, 2003).

Review sessions are vital for training students in the development of their creativity, and for supervising their progress along the process. A main goal of the review sessions is to exchange feedback between students and instructors. In the current study review sessions are considered as convenient environments for investigating the interactions maintained between the parties.

The type of information involved in such communication process (Uloglu, 2000) is supposed to affect the creativity of the design outcome. However, what type of information is generated and communicated during these interactions, and how it may enhance the different aspects of design creativity has yet to be addressed. Therefore, this work will explore such communications in order to analyse the semantic content of review sessions, and its relation to the creativity of the design outcomes.

\section{Semantic analysis in design}

The semantic nature of verbalisations represents a fundamental resource to categorize, quantify and compare the information generated and conveyed through interactions. Semantic analysis approach can 
assist to this aim by describing human thinking as a network where a concept can lead to several related concepts. In design problem solving, semantic analysis enables the representation, quantification and modelling of idea generation (Georgiev et al., 2010; Taura et al., 2012; Georgiev and Taura, 2014; Yamamoto et al., 2009), and other mental processes (Cash et al., 2014). Furthermore, semantic approaches based on natural language processing such as lexical chain analysis were used to differentiate discontinuities in agreement in design problem solving (Dong, 2009). However, not many of these methods investigate design creativity, specifically.

In order to bridge the existing research gap, this study employs an alternative semantic analysis approach to the classic ones (Dong, 2009). The approach is based on the use of quantifiable measures of semantic networks aimed at representing fundamental cognitive processes in design. A characteristic of these networks is that it enables the measurement and comparison of verbal information. In this study, four semantic measures are used to quantify fundamental process involved in design problem solving, includes: Polysemy, Abstraction, IC, and Semantic Similarity that are described as follows.

Polysemy can be defined as the quality of a word that have multiple meanings. Polysemy is identified as a critical manifestation of the flexibility and adaptability in meaning potential (Fauconnier and Turner, 2003). Abstraction is defined as a generalisation from specific instances that have a higher level of detail in information, implying a simplification of more essential features. It is known that abstract compared to specific ways of thinking lead to novel and open-ended ideas (Ward et al., 2004). Information Content (IC) is defined as the amount of information transmitted by a specific unit of language in certain context. Units with higher IC have a lower probability of occurrence. IC was found to be effective to quantify design fixation during idea generation (Gero, 2011). Most typical measures used in natural language processing are those concerned with Semantic Similarity (e.g., Resnik, 1995), measuring how equal are two words, and how thoroughly they represent human similarity judgements. Recent research works centred on semantic analysis and design creativity processes. For example, Taura et al. (2012) showed that Polysemy correlates significantly with the originality of the new design ideas generated from two given initial concepts. Moreover, Semantic Similarity was successfully used to quantify the degree of convergence and divergence in design thinking (Georgiev and Georgiev, 2018). The novelty of the resulting combination of two concepts was also found to be related to semantic similarity (Nomaguchi et al., 2019). Semantic networks are used in computational tools aiming at enhancing the fluency of idea generation (Han et al., 2018).

Notwithstanding these works, design literature on semantic networks and creativity is scarce, and therefore the current study aims to extend research in this direction. Major advantages of using semantic networks for analysing conversations are: i) the applicability of the method for exploring cognitive processes, and ii) the robust computation of objective information theory measures (i.e., Abstraction, Polysemy, IC and Semantic Similarity) to quantify these processes. It is expected that the semantic measures will be useful in analysing the content of the design conversations, and in understanding their relation to the creativity of the design outcomes. It is also proposed that differences will be found between students and instructors on these relationships.

\section{Method}

The analysed data is based on 35 design review conversations from the 10th Design Thinking Research Symposium (DTRS10) set (Adams and Siddiqui, 2014). Six junior and six graduate students, all majoring in Industrial Design, took part in the design sessions, in addition to two design teachers and 16 guest professional experts who participated as instructors. The meetings took place during different critique sessions carried out between students and instructors. Design sessions were video-taped and transcribed as a part of the dataset. The purpose of the design sessions was to provide critique to develop a solution aimed for a real client. Sessions lasted about 15 minutes. The task for the junior students dealt with the design of "Impromptu" seating places, supporting collaborative work environments (See Figure 1). For the graduate students, the task consisted in designing an "Outside Laundry Room" place aimed at enhancing the laundry experience.

The assessment of the creativity metrics was carried out by two independent referees, who used a 1 to 5 value Likert scale. They evaluated the design outcomes by means five creativity variables described in Section 2 dealing with Originality, Usability, Feasibility, Overall Value, and Overall Creativity. 
Definitions for each of these variables were given to the referees as part of the assessment criteria: Comparted to Originality (i.e, how dissimilar a solution is from standard solutions in the context of this study), Usability (i.e., efficiency, performance, and response to practical needs) and Feasibility (i.e., technology/materiality), the metrics of Overall Value (i.e., worthiness compared to standard solutions) and Overall Creativity (based on Amabile's (1996) Consensual Assessment Technique - a measurement tool where expert evaluators assess the general creativity of designs according to their knowledge) might be seen as relatively less objective for assessing the final design outcomes. Although Overall Value may overlap to some extent with Overall Creativity, both metrics were included in this study since they offer a more comprehensive criteria that is easy to understand by lay persons.

Cohen's kappa coefficient (Cohen, 1988) was used to establish the level of agreement between the two referees on their assessments of the creativity of the final solutions. Table 1 shows that there was a substantial and significant agreement for all the assessed variables.

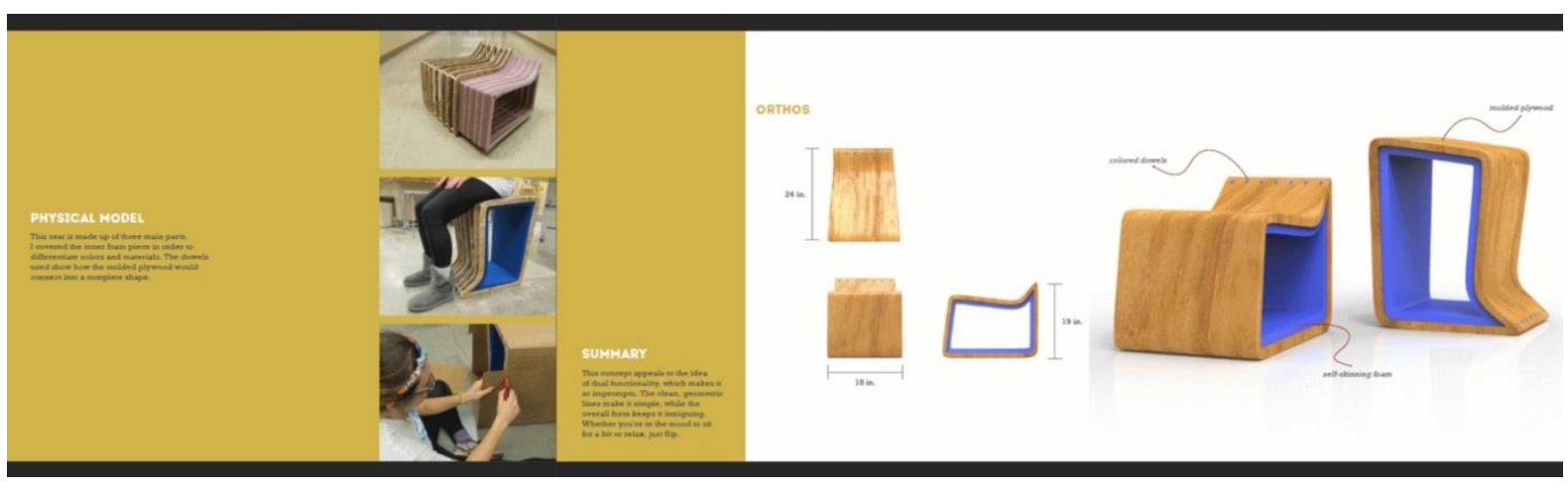

Figure 1. Example of a design outcome by a junior student

Table 1. Cohen's Kappa k evaluation of agreement between the two referees

\begin{tabular}{|c|c|c|c|c|c|}
\hline & Original & Usable & Feasible & Overall Value & Overall Creativity \\
\hline Kappa & 0.883 & 0.780 & 0.885 & 0.644 & 0.872 \\
\hline Sig. & 0.000 & 0.000 & 0.000 & 0.000 & 0.000 \\
\hline
\end{tabular}

For the sake of automating the measure of semantic values, standard natural language processing tools were used to extract nouns from the design conversations. The four semantic measures of Polysemy, Abstraction, Information Content and Semantic Similarity were calculated as average values of all the conversations maintained by each student (see Georgiev and Georgiev, 2018). Calculations were based on existing graph-theoretic and information-theoretic formulas (Resnik, 1997; Blanchard, 2008).

The semantic approach included the following steps: First, the textual data was processed using partof-speech tagging with the Natural Language Toolkit (NLTK) (Bird et al., 2009). Thereafter, only singular and plural nouns were extracted. Finally, all the identified nouns were processed by converting plurals into singular forms, and by discarding those nouns that were not listed in the WordNet database. WordNet is a database represented as a knowledge structure in the form of a hierarchical and interconnected network of words, which is available online.

\section{Results}

\subsection{Creativity variables and semantic measures in design conversations}

For the sake of exploring the relation between the four semantic measures and the five creativity measures a correlation analysis was carried out, and p-values were reported (See Table 2). The results showed significant correlations between Feasibility and Polysemy, Abstraction and IC measures. Significant correlations were also found between IC and Originality, Feasibility, and Overall Creativity. Finally, Overall Value was observed to correlate with Similarity. 


\subsection{Creativity variables and semantic measures in verbalizations of instructors and students}

In order to explore the individual verbalizations of students and instructors in the design conversations, the relations between the four semantic measures and the five creativity variables were analysed separately for each party. The four semantic measures were calculated per verbalizations of instructors and students. Correlation analyses were carried out and p-values were reported (See Table 3). For the instructors, results showed significant correlations between Feasibility and Polysemy, Abstraction and IC measures. Significant correlations were also found between IC and Originality, Feasibility, and Overall Creativity. In the case of the students, Similarity was observed to correlate with Overall Value.

Table 2. Spearman correlations between semantic measures and creativity variables

\begin{tabular}{|c|c|c|c|c|c|c|}
\hline \multicolumn{2}{|c|}{ Spearman correl. } & Originality & Usability & Feasibility & Overall Value & Overall Creativity \\
\hline \multirow{2}{*}{ Polysemy } & Corr. & 0.015 & $.618^{*}$ & $.615^{*}$ & 0.471 & -0.100 \\
\cline { 2 - 7 } & Sig. & 0.964 & 0.032 & 0.033 & 0.122 & 0.757 \\
\hline \multirow{2}{*}{ Abstraction } & Corr. & -0.410 & 0.135 & $.860^{* *}$ & 0.014 & -0.404 \\
\cline { 2 - 7 } & Sig. & 0.185 & 0.676 & 0.000 & 0.965 & 0.193 \\
\hline \multirow{2}{*}{ IC } & Corr. & $.657^{*}$ & -0.213 & -0.471 & 0.176 & $.800^{* *}$ \\
\cline { 2 - 7 } & Sig. & 0.020 & 0.506 & 0.122 & 0.584 & 0.002 \\
\hline \multirow{2}{*}{$\begin{array}{c}\text { Semantic } \\
\text { Similarity }\end{array}$} & Corr. & 0.076 & 0.490 & 0.450 & $.695^{*}$ & -0.152 \\
\cline { 2 - 7 } & Sig. & 0.814 & 0.106 & 0.142 & 0.012 & 0.638 \\
\hline
\end{tabular}

Table 3. Spearman correlations between semantic measures and creativity evaluations for instructors and students

\begin{tabular}{|c|c|c|c|c|c|c|}
\hline \multicolumn{9}{|c|}{ Instructors } \\
\hline Spearman correlation & Originality & Usability & Feasibility & Overall Value & Overall Creativity \\
\hline \multirow{3}{*}{ Polysemy } & Corr. & -0.098 & 0.526 & $.749^{* *}$ & 0.291 & -0.089 \\
\cline { 2 - 7 } & Sig. & 0.762 & 0.079 & 0.005 & 0.358 & 0.784 \\
\hline \multirow{2}{*}{ Abstraction } & Corr. & -0.007 & 0.316 & $.605^{*}$ & 0.209 & -0.019 \\
\cline { 2 - 7 } & Sig. & 0.982 & 0.317 & 0.037 & 0.515 & 0.954 \\
\hline \multirow{2}{*}{ IC } & Corr. & 0.178 & -0.306 & $-.626^{*}$ & 0.007 & 0.226 \\
\cline { 2 - 7 } & Sig. & 0.580 & 0.334 & 0.029 & 0.982 & 0.480 \\
\hline \multirow{2}{*}{ Semantic } & Corr. & -0.098 & 0.522 & $.698^{*}$ & 0.360 & -0.133 \\
\cline { 2 - 7 } Similarity & Sig. & 0.762 & 0.082 & 0.012 & 0.251 & 0.680 \\
\hline \multirow{3}{*}{ Spearman correlation } & Originality & Usability & Feasibility & Overall Value & Overall Creativity \\
\hline \multirow{2}{*}{ Polysemy } & Corr. & -0.084 & 0.227 & 0.489 & 0.504 & -0.196 \\
\cline { 2 - 7 } & Sig. & 0.796 & 0.477 & 0.106 & 0.095 & 0.541 \\
\hline \multirow{2}{*}{ Abstraction } & Corr. & -0.414 & 0.220 & 0.453 & 0.234 & -0.541 \\
\cline { 2 - 7 } & Sig. & 0.181 & 0.491 & 0.139 & 0.464 & 0.070 \\
\hline \multirow{2}{*}{ IC } & Corr. & 0.247 & 0.011 & -0.036 & -0.176 & 0.344 \\
\cline { 2 - 7 } & Sig. & 0.439 & 0.974 & 0.912 & 0.584 & 0.273 \\
\hline \multirow{2}{*}{ Semantic } & Corr. & 0.185 & 0.409 & 0.155 & $.723^{* *}$ & -0.026 \\
\cline { 2 - 7 } Similarity & Sig. & 0.564 & 0.187 & 0.631 & 0.008 & 0.936 \\
\hline
\end{tabular}

\section{Discussion}

\subsection{Creativity and semantic measures in design interactions}

Findings showed that IC was significantly related to the Originality of the final solutions, as demonstrated by the correlation analysis. Moreover, IC was also found to be strongly related to Overall Creativity. These findings are in line with Gero (2011), who showed that a sharp drop in IC was related 
to design fixation. Georgiev and Georgiev (2018) also observed that with an increase in IC, the generation of successful ideas - known to lead to creative outcomes, was also increased.

While no previous study focused on the relation between Semantic Similarity and Originality, Georgiev and Georgiev (2018) also found that Semantic Similarity decreasing in time was associated with the generation of successful ideas. The present findings should not necessarily contradict previous ones, but rather they provide additional evidence to the importance of Semantic Similarity to design creativity. With the exception of IC, Overall Creativity was not found to be related to the other semantic measures. However, previous studies found significant relations between Creativity (measured as the originality of the produced ideas), Polysemy (Taura et al., 2012), and Abstraction (Ward et al., 2004). Considering that IC can be defined as the inverse probability of ordinary language occurrence, it is proposed that domain-specific language can be employed in support of the generation of Original and creative outcomes.

Additionally, it was observed that feasible solutions are the outcome of design conversations involving high levels of Polysemy and Abstraction. It is suggested that being fluent in abstract words might contribute to produce design outcomes that can be materialized in real practice. No previous studies connecting Feasibility with semantic measures such as Abstraction were found in literature. Nevertheless, the nearest ones indicate that a relation between Creativity, Polysemy, and Abstraction (Taura et al., 2012; Ward et al., 2004) may exist. Building on these findings, recommendations can be proposed as instructional strategies to be considered when the pedagogical goal is to produce feasible solutions. For example, information exchange can be characterized by common language and the use of generalizations.

Polysemy was related to Usability. Hence, Polysemy plays a role regarding the functionality of the design outcome, which is in line with previous studies (Taura et al., 2012; Georgiev and Taura, 2014). Semantic Similarity was found to be related with the Overall Value of the design outcomes. This finding suggests that when the Semantic Similarity of the conversations are increased, the Overall Value of the design outcomes also increases. Semantic Similarity was positively related to the self-perceived assessment of the quality of the design outcomes (Georgiev et al., 2008). Hence, it is proposed that employing instances that are close one another can enhance the chances that designers working in a specific context would perceive a design outcome as having higher added value and possibly being more original.

\subsection{Creativity and semantic measures: instructors and students}

In order to elaborate further upon the individual contribution of students and instructors to the design conversations, the relations among the four semantic measures and the five creativity variables are discussed separately for each party. In general, results showed that in the case of instructors, semantic measures were related to Feasibility, whereas for students a relation was observed with the Overall Value of the solution. A possible reason for these is that, due to the nature of the design task, students mainly focused on concepts and ideas, at the time that instructors were more concrete and practically oriented.

It is also remarkable that the semantic measures generated either by students or instructors were neither associated with Originality, nor with the Overall Creativity of the design. These contrast with a previous analysis of the exchanges between students and instructors, where IC was found to predict Originality. It is proposed that the co-work of the two parties played a fundamental role in enhancing the novelty of the final solution.

Moreover, for instructors, it was observed that design conversations involving high levels of Polysemy, Abstraction, and Semantic Similarity, and low levels of IC were significantly related to design outcomes characterized by Feasibility. It is possible that instructors, who were very interested in helping students to produce feasible solutions, alternated the use of the four semantic measures in order to transmit knowledge clearly and efficiently.

In the case of the students, Semantic Similarity was found to be significantly related to the Overall Value of the design solutions. It is proposed that the use of alike terms helped them to expand their conceptual jargon from known to less familiar related terms. It is possible that using such substitute related terms played a role to produce less standard and more valuable solutions. On the other hand, no 
significant relations were observed among the remaining creativity variables and the semantic measures. Thus, it is likely that students, who do not possess strongly developed structures of knowledge, had a rather modest input to enrich the conversation through the design sessions. In the overall picture of the role of the students in design conversations, previous studies pointed out students' selection of conventional ideas (Starkey et al., 2016).

\section{Conclusions}

In this study we investigated the validity and benefit of employing a semantic approach to analyse design conversations in the studio, and its relation to design creativity. For this purpose, Polysemy, Abstraction, Information Content (IC) and Semantic Similarity were used as major semantic measures. These were easy to compute, and helped to gain a better understanding about the nature of design verbalizations by students and instructors. There are few semantic network approaches in the literature, most of which are computationally costly. An advantage of the present approach is that it is simpler than these, and therefore its implementation demands less statistical and analytical effort. For these reasons, it can be employed in real time for a variety of purposes such as the analysis and support of information exchange.

Moreover, the semantic approach showed to be valid for analysing and capturing the semantic content of the different dialogues, as well as for exploring the relevance of such content for design creativity. Moreover, semantic measures were observed to have a dissimilar contribution to design creativity. Accordingly, these enhanced different aspects of creativity. On the other hand, findings unveiled a significant relationship between IC and Originality and Overall Creativity suggesting that designers fluent in rich lexicon might be highly creative, and capable of producing innovative outcomes.

It is also remarkable that when verbalizations were analysed individually, instructors and students showed to have a different input to the creativity of the outcomes. Semantic measures of the instructors were mainly related to Feasibility, whereas for students the focus was set on the Overall Value. These suggest that the personal goals of designers regarding the creativity aspects that they seek to foster may have an impact on the type of language used during the conversations. Intervention programs interested in promoting design creativity can benefit from implementing the present findings in the design studio. For example, knowledge-based systems aimed at identifying semantic content in the verbalizations by students and instructors, can be employed in real-time during design conversations. The use of systems of this kind can serve to provide instant feedback about their individual contribution to design creativity. The paper should be seen as an explorative work based on a small sample of participants. Hence, rather than focusing on the research findings themselves, we were more interested in gaining insight upon the approach and its significance for design creativity. A future study will include a larger sample of participants with different levels of knowledge and expertise, belonging to other design disciplines. The current findings reflect the way that teachers compared to students use semantic measures during the design process. However, dissimilar results might be obtained when the sample includes designers working in real practice. This will be explored in a future work extending the present study.

Acknowledgements

The authors would like to thank Dr. Robin Adams for allowing access to the DTRS Database (Adams, 2015) and Dr. Danko D. Georgiev for the help in the semantic calculations. This research has been partially financially supported by Academy of Finland 6Genesis Flagship (grant 318927).

\section{References}

Adams, R.S. (2015), "Design review conversations: The dataset”, In: Adams, R. S. and Siddiqui, J. A., (Eds.), Analyzing Design Review Conversations, West Lafayette, Indiana: Purdue University Press.

Adams, R.S. and Siddiqui, J.A. (2013), "Purdue DTRS - Design Review Conversations Database", XRoads Technical Report, TR-01-13, West Lafayette, Indiana: Purdue University.

Amabile, T. (1996), "Creativity in Context: Update to the Social Psychology of Creativity", Boulder, CO: Westview.

Blanchard, E., Harzallah, M. and Kuntz, P. (2008), "A generic framework for comparing semantic similarities on a subsumption hierarchy", In: Ghallab, M., Spyropoulos, C.D., Fakotakis, N. and Avouris, N. (Eds.), ECAI 2008: 18th European Conference on Artificial Intelligence including Prestigious Applications of Intelligent Systems (PAIS 2008), IOS Press, Patras, Greece, pp. 20-24. 
Cash, P., Stanković, T. and Štorga, M. (2014) "Using visual information analysis to explore complex patterns in the activity of designers", Design Studies, Vol. 35 No. 1, pp. 1-28. http://dx.doi.org/10.1016/j.destud.2013.06.001 Christensen, B.T. and Ball, L.J. (2016), "Dimensions of creative evaluation: Distinct design and reasoning strategies for aesthetic, functional and originality judgments", Design Studies, Vol. 45, 116-136. https://doi.org/10.1016/j.destud.2015.12.005

Cohen, J. (1988), "Statistical power analysis for the behavioral sciences (2nd ed.)", New York: Psychology Press. Cross, N. (1983), "The relevance of cognitive styles in design education", Design Methods and Theories, Vol. 17 No.1.

Demirbaş, O.O. and Demirkan, H. (2003), "Focus on architectural design process through learning styles", Design Studies, Vol. 24 No. 5, pp. 437-456. http://dx.doi.org/10.1016/S0142-694X(03)00013-9

Dong, A. (2009), "The Language of Design: Theory and Computation", London: Springer.

Fauconnier, G. and Turner, M. (2003), "Polysemy and Conceptual Blending", In: Nerlich, B., Herman, V., Todd, Z. and Clarke, D., (Eds.), Polysemy: Flexible Patterns of Meaning in Mind and Language, Berlin and New York: Mouton de Gruyter, pp. 79-94.

Georgiev, G.V. and Georgiev, D.D. (2018), "Enhancing User Creativity: Semantic Measures for Idea Generation", Knowledge-Based Systems, Vol. 151, pp. 1-15. http://dx.doi.org/10.1016/j.knosys.2018.03.016

Georgiev, G.V., Nagai, Y. and Taura, T. (2008), "Method of design evaluation focused on relations of meanings for a successful design", Tenth International Design Conference Design 2008, May 19-22, Dubrovnik, Croatia, pp. 1149-1158.

Georgiev, G.V., Nagai, Y. and Taura, T. (2010), "A method for the evaluation of meaning structures and its application in conceptual design", Journal of Design Research, Vol. 8 No. 3, pp. 214-234, http://dx.doi.org/10.1504/jdr.2010.032607

Georgiev, G.V. and Taura, T. (2014), "Polysemy in design review conversations", 10th Design Thinking Research Symposium, Purdue University, West Lafayette, Indiana: Purdue University.

Gero, J.S. (2011), "Fixation and commitment while designing and its measurement", The Journal of Creative Behavior, Vol. 45 No. 2, pp. 108-115.

Han, J., Shi, F., Chen, L., \& Childs, P. R. (2018). "The Combinator-a computer-based tool for creative idea generation based on a simulation approach". Design Science, 4, e11. http://dx.doi.org/10.1017/dsj.2018.7

Kreitler, S. and Casakin, H. (2009), "Self-perceived creativity: The perspective of design", European Journal of Psychological Assessment, Vol. 25 No. 3, pp. 194-203. http://dx.doi.org/10.1027/1015-5759.25.3.194

Nomaguchi, Y., Kawahara, T., Shoda, K., \& Fujita, K. (2019). Assessing Concept Novelty Potential with Lexical and Distributional Word Similarity for Innovative Design. In Proceedings of the Design Society: International Conference on Engineering Design (Vol. 1, No. 1, pp. 1413-1422). Cambridge University Press. http://dx.doi.org/10.1017/dsi.2019.147

Resnik, P. (1995), "Using Information Content to evaluate Semantic Similarity in a taxonomy", IJCAI'95 Proceedings of the 14th international joint conference on Artificial intelligence - Volume 1: Morgan Kaufmann Publishers, pp. 448-453.

Siang, J.K.K., Chia, P.Z., Koronis, G. and Silva, A. (2018), "Exploring the use of a full factorial design of experiment to study design briefs for creative ideation", ASME 2018 International Design Engineering Technical Conferences and Computers and Information in Engineering Conference, Quebec City, Quebec, Canada, August 26-29, V007T06A008-V007T06A008, http://dx.doi.org/10.1115/DETC2018-85794

Sarkar, P. and Chakrabarti, A. (2011), “Assessing design creativity”, Design Studies, Vol. 32 No. 4, pp. 348-383, http://dx.doi.org/10.1016/j.destud.2011.01.002

Taura, T., Yamamoto, E., Fasiha, M.Y.N., Goka, M., Mukai, F., Nagai, Y. and Nakashima, H. (2012), "Constructive simulation of creative concept generation process in design: a research method for difficult-toobserve design-thinking processes", Journal of Engineering Design, Vol. 23 No. 4, pp. 297-321. http://dx.doi.org/10.1080/09544828.2011.637191

Uluoğlu, B. (2000), "Design knowledge communicated in studio critiques", Design Studies, Vol. 21 No. 1, pp. 33-58. http://dx.doi.org/10.1016/S0142-694X(99)00002-2

Ward, T.B., Patterson, M.J. and Sifonis, C.M. (2004), "The Role of Specificity and Abstraction in Creative Idea Generation", Creativity Research Journal, Vol. 16 No. 1, pp. 1-9. http://dx.doi.org/10.1207/s15326934crj1601_1 Yamamoto, E., Goka, M., Yusof, M., Fasiha, N., Taura, T. and Nagai, Y., (2009), "Virtual modeling of concept generation process for understanding and enhancing the nature of design creativity", In: DS 58-2: Proceedings of ICED 09, the 17th International Conference on Engineering Design, Vol. 2, Design Theory and Research Methodology, Palo Alto, CA, USA, August 24-27. 